\title{
ON THE ARITHMETIC-GEOMETRIC MEAN INEQUALITY
}

\author{
MEHDI HASSANI
}

\begin{abstract}
We obtain some refinements of the Arithmetic-Geometric mean inequality. As an application, we find the maximum value of a multi-variable function.
\end{abstract}

\section{Introduction}

We assume that $a_{1}, a_{2}, \ldots, a_{n}$ are $n$ positive real numbers, and as usual, we define their arithmetic and geometric means, respectively by

$$
A=\frac{1}{n} \sum_{i=1}^{n} a_{i}, \quad \text { and } \quad G=\left(\prod_{i=1}^{n} a_{i}\right)^{\frac{1}{n}} .
$$

We consider the functions $g(x)=\mathrm{e}^{x}-x^{\mathrm{e}}$ and $h(x)=x^{1 / x}$ over $(0, \infty)$. The function $h$ has an absolute maximum at $x=\mathrm{e}$. Thus, if $x>0$ then $\mathrm{e}^{1 / \mathrm{e}} \geqslant x^{1 / x}$, or equivalently $g(x) \geqslant 0$, with equality if and only if $x=\mathrm{e}$. For $i=1,2, \ldots, n$, we take $x=a_{i} \mathrm{e} / G$ in $\mathrm{e}^{x} \geqslant x^{\mathrm{e}}$, and then we multiply the resulting inequalities to get

$$
\mathrm{e}^{\frac{\mathrm{e}}{G} n A}=\mathrm{e}^{\frac{\mathrm{e}}{G} \sum_{i=1}^{n} a_{i}} \geqslant\left(\prod_{i=1}^{n} \frac{a_{i} \mathrm{e}}{G}\right)^{\mathrm{e}}=\left(\frac{\mathrm{e}^{n} G^{n}}{G^{n}}\right)^{\mathrm{e}}=\mathrm{e}^{n \mathrm{e}},
$$

from which we obtain $A \geqslant G$, with equality if and only if $a_{i} \mathrm{e} / G=\mathrm{e}$ for $i=1,2, \ldots, n$, or equivalently for when $a_{1}=a_{2}=\cdots=a_{n}$.

The above argument for obtaining the Arithmetic-Geometric mean inequality is due to Schaumberger [1]. In this note we replace $g(x)$ by a smaller positive function to get some refinements of the this inequality. More precisely, we obtain the following result.

Theorem 1.1. Assume that $a_{1}, a_{2}, \ldots, a_{n}$ are $n$ positive real numbers with arithmetic and geometric means $A$ and $G$, respectively. Then, we have

$$
A \geqslant G+\mathscr{R} \geqslant G,
$$

Received May 15, 2013, accepted July 16, 2013.

Communicated by Chung-Tsun Shieh.

2010 Mathematics Subject Classification. 26D15.

Key words and phrases. Arithmetic-Geometric mean inequality. 
where

$$
\mathscr{R}=\frac{G}{n \mathrm{e}} \log \left(1+\frac{1}{\mathrm{e}^{n \mathrm{e}}} \prod_{i=1}^{n}\left(\frac{a_{i}}{G}-1\right)^{2}\right) \geqslant 0,
$$

with equality if and only if $a_{1}=a_{2}=\cdots=a_{n}$.

\section{Proof of Theorem 1.1}

Lemma 2.1. For $x>0$ we define

$$
f(x)=\mathrm{e}^{x}-x^{\mathrm{e}}-\frac{1}{\mathrm{e}^{2}}(x-\mathrm{e})^{2} .
$$

The inequality $f(x) \geqslant 0$ is valid for $x>0$, with equality if and only if $x=\mathrm{e}$. Moreover, $\frac{1}{\mathrm{e}^{2}}$ is the best possible constant for which the above inequality is valid.

Proof. As Figure 1 shows, $f(x)$ takes its minimum value equal to 0 at $x=\mathrm{e}$. Also, we have $\lim _{x \rightarrow 0^{+}} f(x)=0$, which proves optimal choose of the constant $\frac{1}{\mathrm{e}^{2}}$. This completes the proof.
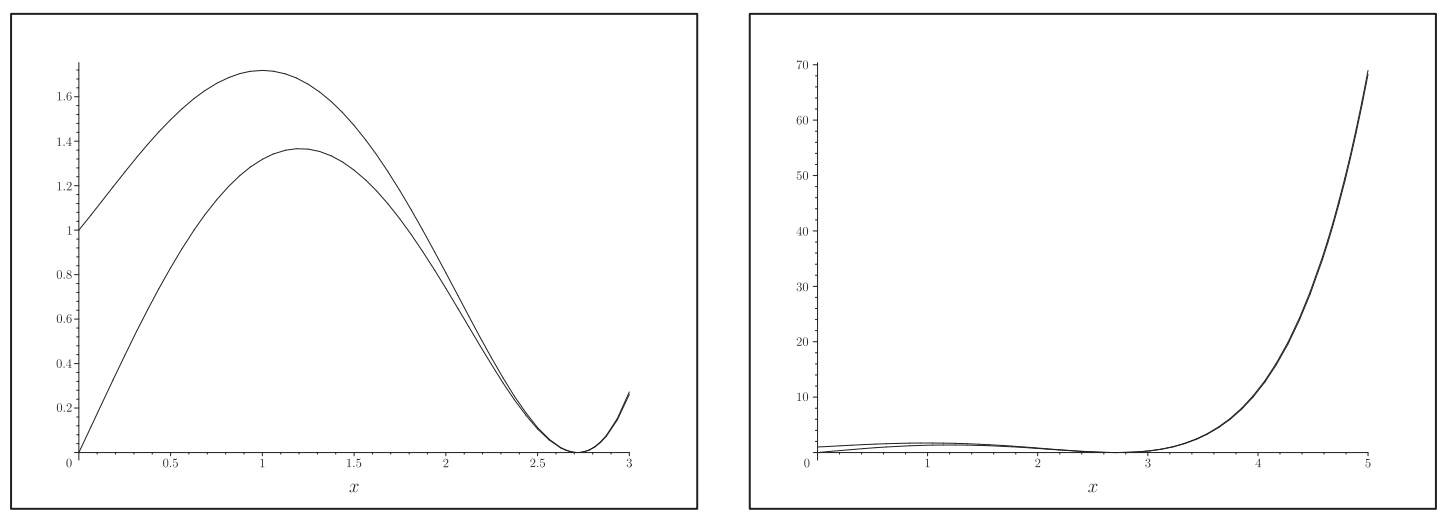

Figure 1: Graphs of the functions $f(x)=\mathrm{e}^{x}-x^{\mathrm{e}}-\frac{1}{\mathrm{e}^{2}}(x-\mathrm{e})^{2}$ and $g(x)=\mathrm{e}^{x}-x^{\mathrm{e}}$ over the intervals $(0,3)$ and $(0,5)$.

Proof of Theorem 1.1. We apply Lemma 2.1 by taking $x=a_{i} \mathrm{e} / G$ in $f(x)$, from which we obtain

$$
\mathrm{e}^{\frac{\mathrm{e}}{G} a_{i}} \geqslant\left(\frac{a_{i} \mathrm{e}}{G}\right)^{\mathrm{e}}+\frac{1}{\mathrm{e}^{2}}\left(\frac{a_{i} \mathrm{e}}{G}-\mathrm{e}\right)^{2}=\left(\frac{a_{i} \mathrm{e}}{G}\right)^{\mathrm{e}}+\left(\frac{a_{i}}{G}-1\right)^{2} \quad(\text { for } i=1,2, \ldots, n) .
$$

We multiply these inequalities to get

$$
\mathrm{e}^{\frac{\mathrm{e}}{G} n A}=\mathrm{e}^{\frac{\mathrm{e}}{G} \sum_{i=1}^{n} a_{i}} \geqslant\left(\prod_{i=1}^{n} \frac{a_{i} \mathrm{e}}{G}\right)^{\mathrm{e}}+\prod_{i=1}^{n}\left(\frac{a_{i}}{G}-1\right)^{2}=\mathrm{e}^{n \mathrm{e}}+\prod_{i=1}^{n}\left(\frac{a_{i}}{G}-1\right)^{2} .
$$

Thus, we have

$$
\mathrm{e}^{\frac{\mathrm{e}}{G} n A} \geqslant \mathrm{e}^{n \mathrm{e}}\left(1+\frac{1}{\mathrm{e}^{n \mathrm{e}}} \prod_{i=1}^{n}\left(\frac{a_{i}}{G}-1\right)^{2}\right) .
$$


Finally, we take logarithm and we divide the resulting inequality by ne to obtain

$$
\frac{A}{G} \geqslant 1+\frac{1}{n \mathrm{e}} \log \left(1+\frac{1}{\mathrm{e}^{n \mathrm{e}}} \prod_{i=1}^{n}\left(\frac{a_{i}}{G}-1\right)^{2}\right),
$$

with equality if and only if $a_{1}=a_{2}=\cdots=a_{n}$. This completes the proof.

\section{Some applications}

On may rewrite the Arithmetic-Geometric mean inequality in the forms

$$
A-G \geqslant 0, \quad \text { and } \quad \frac{A}{G}-1 \geqslant 0 .
$$

As the first application of Theorem 1.1, we obtain the following refinement of the above mentioned inequalities.

Theorem 3.1. Assume that $a_{1}, a_{2}, \ldots, a_{n}$ are $n$ positive real numbers which are not simultaneously equal, with arithmetic and geometric means $A$ and $G$, respectively. Then, we have

$$
A-G \geqslant \frac{G \mathrm{e}^{n \mathrm{e}\left(\frac{A}{G}-1\right)}}{n \mathrm{e}\left(\mathrm{e}^{n \mathrm{e} \frac{A}{G}}-\mathrm{e}^{n \mathrm{e}}\right)} \prod_{i=1}^{n}\left(\frac{a_{i}}{G}-1\right)^{2} \geqslant 0,
$$

or equivalently

$$
\frac{A}{G}-1 \geqslant \frac{\mathrm{e}^{n \mathrm{e}\left(\frac{A}{G}-1\right)}}{n \mathrm{e}^{n \mathrm{e}+1}\left(\mathrm{e}^{n \mathrm{e}\left(\frac{A}{G}-1\right)}-1\right)} \prod_{i=1}^{n}\left(\frac{a_{i}}{G}-1\right)^{2} \geqslant 0 .
$$

Proof. Assume that $a_{1}, a_{2}, \ldots, a_{n}$ are $n$ positive real numbers which are not simultaneously equal, so that $A>G$. By using the result of Theorem 1.1, we have $\mathscr{R} \leq A-G$, which is equivalent to

$$
\frac{1}{\mathrm{e}^{n e}} \prod_{i=1}^{n}\left(\frac{a_{i}}{G}-1\right)^{2} \leqslant \mathrm{e}^{n \mathrm{e}\left(\frac{A}{G}-1\right)}-1 .
$$

On the other hand, for $0 \leqslant x \leqslant \beta$, we have $\log (1+x) \geqslant \frac{\log (1+\beta)}{\beta} x$ because $\frac{\log (1+x)}{x}$ is decreasing on $(0, \beta]$. We use this inequality by putting $x=\frac{1}{\mathrm{e}^{n e}} \prod_{i=1}^{n}\left(\frac{a_{i}}{G}-1\right)^{2} \geqslant 0$ and $\beta=\mathrm{e}^{n \mathrm{e}\left(\frac{A}{G}-1\right)}-1$ to get

$$
\mathscr{R}=\frac{G}{n \mathrm{e}} \log \left(1+\frac{1}{\mathrm{e}^{n \mathrm{e}}} \prod_{i=1}^{n}\left(\frac{a_{i}}{G}-1\right)^{2}\right) \geqslant \frac{G}{n \mathrm{e}}\left(\frac{\mathrm{e}^{n \mathrm{e}\left(\frac{A}{G}-1\right)}}{\mathrm{e}^{n \mathrm{e} \frac{A}{G}}-\mathrm{e}^{n \mathrm{e}}} \prod_{i=1}^{n}\left(\frac{a_{i}}{G}-1\right)^{2}\right) .
$$

This completes the proof.

As the second application of Theorem 1.1, we observe that it allows us to find the maximum value of a multi-variable function, without using partial derivative tests. 
Theorem 3.2. We have

$$
\max _{a_{i}>0} \frac{G}{n \mathrm{e}} \log \left(1+\frac{1}{\mathrm{e}^{n \mathrm{e}}} \prod_{i=1}^{n}\left(\frac{a_{i}}{G}-1\right)^{2}\right)=A-G .
$$

Remark 3.3. We assume that $a_{i}>0$, and then we replace $a_{i}$ by $1 / a_{i}$, from which the inequality $A \geqslant G$ implies validity of the well-known Geometric-Harmonic mean inequality, asserting that $G \geqslant H$, where $H$ refers to the harmonic mean of the positive real numbers $a_{1}, a_{2}, \ldots, a_{n}$. We observe that the replacement $a_{i} \rightarrow 1 / a_{i}$ gives the replacements $A \rightarrow 1 / H$ and $G \rightarrow 1 / G$. By applying this fact, one may rewrite all of the above results concerning the means $A$ and $G$, to obtain similar results concerning the means $G$ and $H$.

\section{Acknowledgement}

I express my gratitude to the referee for giving useful comments.

\section{References}

[1] N. Schaumberger, The AM-GM Inequality via $x^{1 / x}$, College Math. J., 20(1989), p320.

Department of Mathematics, University of Zanjan, University Blvd., 45371-38791, Zanjan.

E-mail: mehdi.hassani@znu.ac.ir 